\title{
The Value of an Appreciation of Clinical Profiles in Communities as Aide-mémoire When Faced with Difficult Ophthalmic Diagnoses
}

Ophthalmologists and especially subspecialists tend to focus attention on the organ or the subsection of the eye with often very little consideration to the rest of the body and even less to the economic, geographic, cultural, demographic, and social determinants of health and wellness of the individual patient and the community. The latter is often left to academics and health authorities to be concerned about. Often this lack of concern with the "bigger picture" results in suboptimal clinical diagnostic accuracy.

When a physician practises in a community it is essential to obtain a knowledge of the disease profiles of the particular community or country. For instance, it is well appreciated that certain ophthalmic conditions rarely occur in Black African people. Examples include basal cell carcinoma of the eyelids, malignant melanoma of the choroid, age-related macular degeneration, and multiple sclerosis. On the contrary, the World Health Organization reports that in 2015, 7 million people in South Africa lived with HIV infection, translating to a prevalence of $19.2 \%$ among individuals aged 15 years and older. During 2015, 380,000 new infections were reported and 180,000 deaths were attributed to AIDS during the same period. ${ }^{[1]}$ Furthermore, of the 9.6 million people who in 2014 contracted TB worldwide, $28 \%$ were in Africa. The incidence of TB on the continent of Africa is 281 cases per 100,000 inhabitants compared to the global average of 133 cases per $100,000 .{ }^{[2]}$

An appreciation of the above disease prevalence profiles in the African setting will assist the clinician ophthalmologist to intelligently pursue a diagnostic path in an individual patient with a particular condition.

In the paper by Mustak and Cook in this issue, entitled "Clinical Profile and Outcomes of Optic Neuritis in an HIV Prevalent Urban Community in South Africa" they found that optic neuritis in their community differs from that encountered in first world affluent European and North American countries. In the indigent population served by their hospital, it was found that the majority of patients who presented with optic neuritis either had a secondary cause or presented with atypical features. They also found that HIV and syphilis accounted for most of the secondary causes of atypical optic neuritis.
This knowledge is invaluable in assessing patients with atypical optic neuritis when a clinician practises in their setting.

In a recent study also in South Africa, Laaks et al. analysed aqueous polymerase chain reaction (PCR) results in 72 idiopathic uveitis cases to determine the prevalence of 6 different herpes viridae. They had a $47.2 \%$ positive PCR yield with statistically significant correlation between a positive yield and being HIV $+(P=0.018)$; between an Epstein-Bar virus + yield and being HIV $+(P=0.026)$ and a Cytomegalovirus + result and being HIV $+(P=0.032) \cdot{ }^{[3]}$ A knowledge of the HIV prevalence in the community, therefore, assists greatly in enhancing diagnostic accuracy.

Similarly, Smit et al. reported the prevalence of intraocular tuberculosis in a prospective South African study in a tertiary Eye Clinic. Thirty-five of 106 patients with uveitis $(33.0 \%)$ were diagnosed with intraocular tuberculosis of which $11(31.4 \%)$ had HIV infection. This is much higher than in affluent first world countries. The knowledge that South Africa has a high prevalence of tuberculosis assists the ophthalmologist in suspecting and finally diagnosing intraocular tuberculosis. ${ }^{[4]}$

In as yet unpublished data generated in our hospital in Cape Town, South Africa, we found that $80 \%$ of HIV + cases presenting with uveitis had infectious uveitis, with intraocular tuberculosis, herpetic virus, and syphilis as the most common causes.

Standing back, looking wider than the involved organ, or even the individual person, but doing and communicating quality research on disease prevalence and profiles in communities will assist clinicians immensely in improving diagnostic accuracy in difficult clinical scenarios where we unfortunately still too often revert to the clinical "garbage" basket called "idiopathic." ${ }^{[5]}$

David Meyer Department of Ophthalmology, Faculty of Medicine and Health Sciences, University of Stellenbosch, Cape Town, South Africa 
Address for correspondence: Prof. David Meyer, Department of Ophthalmology, Faculty of Medicine and Health Sciences, University of Stellenbosch, Cape Town, South Africa. E-mail: dm2@sun.ac.za

\section{References}

1. Joint United Nations Programme on HIV/AIDS (UNAIDS). Global AIDS Update; 2016. Geneva, Switzerland; 2016.

2. World Health Organization. Global Tuberculosis Report 2015; World Health Organization. 2015.

3. Laaks D, Smit DP, Harvey J. Polymerase chain reaction to search for Herpes viruses in uveitic and healthy eyes: A South African perspective. Afr Health Sci 2015;15:748-54.

4. Smit DP, Esterhuizen TM, Meyer D. The prevalence of intraocular tuberculosis in HIV-positive and HIV-negative patients in South Africa using a revised classification system. Ocul Immunol Inflamm 2016:1-8. [Epub ahead of print].

5. Meyer D. Are we slowly erasing "idiopathic" from our ophthalmic vocabulary? Middle East Afr J Ophthalmol 2012;19:355-6.
This is an open access article distributed under the terms of the Creative Commons Attribution-NonCommercial-ShareAlike 3.0 License, which allows others to remix, tweak, and build upon the work non-commercially, as long as the author is credited and the new creations are licensed under the identical terms.

\begin{tabular}{|l|l|}
\hline \multicolumn{2}{|c|}{ Access this article online } \\
\hline Quick Response Code: & \\
\hline & Website: \\
\hline & www.meajo.org \\
\cline { 1 - 2 } & \\
\hline
\end{tabular}

How to cite this article: Meyer D. The value of an appreciation of clinical profiles in communities as Aide-mémoire when faced with difficult ophthalmic diagnoses. Middle East Afr J Ophthalmol 2017; $24: 119-20$. 